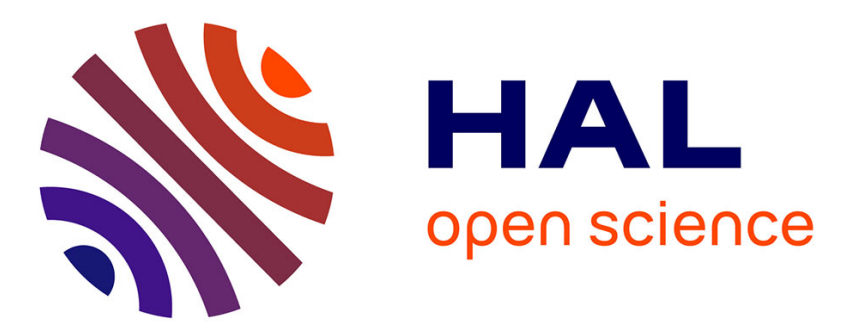

\title{
On Transformation Pathways of General Stress Controlled Thermoelastic Martensitic Transformation in Shape Memory Alloys
}

\author{
P. Sittner, M. Takakura, Y. Hara, M. Tokuda
}

\section{To cite this version:}

P. Sittner, M. Takakura, Y. Hara, M. Tokuda. On Transformation Pathways of General Stress Controlled Thermoelastic Martensitic Transformation in Shape Memory Alloys. Journal de Physique IV Proceedings, 1996, 06 (C1), pp.C1-357-C1-366. 10.1051/jp4:1996134 • jpa-00254166

\section{HAL Id: jpa-00254166 https://hal.science/jpa-00254166}

Submitted on 1 Jan 1996

HAL is a multi-disciplinary open access archive for the deposit and dissemination of scientific research documents, whether they are published or not. The documents may come from teaching and research institutions in France or abroad, or from public or private research centers.
L'archive ouverte pluridisciplinaire HAL, est destinée au dépôt et à la diffusion de documents scientifiques de niveau recherche, publiés ou non, émanant des établissements d'enseignement et de recherche français ou étrangers, des laboratoires publics ou privés. 


\title{
On Transformation Pathways of General Stress Controlled Thermoelastic Martensitic Transformation in Shape Memory Alloys
}

\author{
P. Sittner, M. Takakura*, Y. Hara* and M. Tokuda* \\ Institute of Physics, Czech Academy of Sciences, Prague, Czech Republic \\ * Faculty of Engineering, Mie University, Tsu, Japan
}

\begin{abstract}
The progress of thermoelastic martensitic transformation/MT/ in Cu-Al-ZnMn shape memory alloy/SMA/ polycrystals subjected to nonproportional thermomechanical loadings was investigated by combined tension and torsion of tubular specimens $\left(T \gg A_{f}\right)$. The strain response of SMA polycrystal to various thermomechanical loadings along prescribed stress-temperature paths was examined. It has been shown that the SMA polycrystal can be loaded - unloaded repeatedly by the change of temperature and general combination of external forces of axial load and torque with relatively well defined, path dependent but well reproducible strain path response.
\end{abstract}

\section{INTRODUCTION}

The constitutive description of complex thermomechanical behaviors of polycrystalline SMA, formulated in terms of macroscopic stresses, strains and temperature (equation 1), is required for engineering applications of SMA. The measured external strain, $E_{i j}$, is composed of the elastic strain, $E_{i j}^{e l}$, transformation strain, $E_{i j}^{t r}$, and thermal dilatation strain, $E_{i j}^{t h}$. The problem stands in the formulation of a simple equation to predict the average transformation strains, $E_{i j}^{t r}(t)$, across the volume of transforming polycrystal (2). It is given by local transformation strains of individual martensite variant particles, $\epsilon_{i j}^{t r}(r, t)$, growing or shrinking in the process of thermoelastic MT [1].

$$
\begin{gathered}
E_{i j}=E_{i j}^{e l}+E_{i j}^{t r}+E_{i j}^{t h}=f\left(\Sigma_{i j}(t), T(t)\right) \\
E_{i j}^{t r}(t)=\frac{1}{V} \int_{V} \epsilon_{i j}^{t r}(r, t) d V
\end{gathered}
$$

The thermodynamical theory of MT [2] explains relatively well the uniaxial stress - strain - temperature behaviors of SMA single crystals, undergoing thermoelastic MT, since the external stresses and strains can be directly related with the progress of MT. The progress of the transformation is controlled by the so called thermoelastic criterion - balance of chemical and mechanical forces driving or opposing the motion of martensite variant interfaces, that can be physically well formulated on the atomic level, just at the moving interfaces. However, the process has a tensorial character in nature and, at present, little is known about the interaction of general stress with thermoelastic martensitic transformation, both experimentally and theoretically. The effect of general external stress on the stress induced, first order solid state transformations has been discussed in the frame of thermodynamics by Kato and Pak [3].

Since the engineering applications of SMA require a simple, but reliable estimation of the general macroscopic thermomechanical behaviors of complex polycrystalline SMA, the transformation behavior of SMA polycrystals is now being widely studied micromechanically on the mesoscopic level $[4,5,6,7]$, based on the experimental results obtained on single crystals. The models predict even the behaviors of SMA polycrystal under complex stress states, but reliable experimental data for such conditions are scarce. 


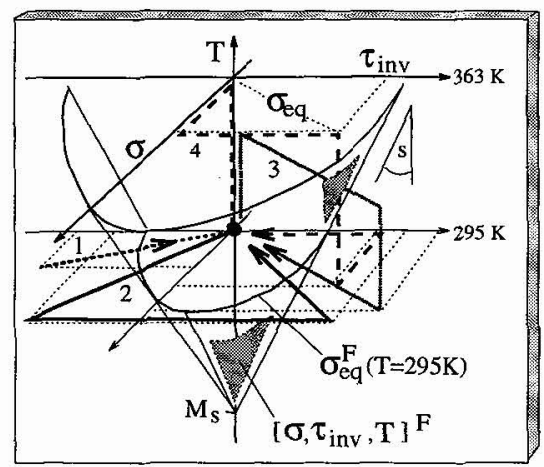

a)

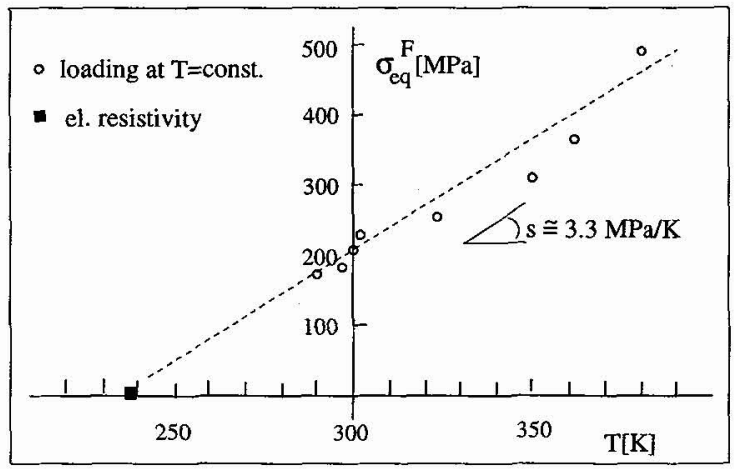

b)

Figure. 1: a) The shape of controlled paths in stress-temperature space: 1-proportional mechanical path, 2-nonproportional mechanical path, 3-proportional thermomechanical path, 4-nonproportional thermomechanical path. b) Temperature dependence of forward transformation start equivalent stress, evaluated on different specimens using first loops in tension, compression, torsion or combined loading

If the pseudoelastic SMA polycrystal is subjected to varying external stress, $\Sigma_{i j}(t)$, and temperature $T(t)$ during a single thermomechanical cycle, the thermoelastic MT takes place and the evolutions of external strain ("strain path") and average transformation strain ("transformation path") characterize the progress of MT on the mesoscopic level of SMA polycrystal. Such transformation paths need not to be identical in forward and reverse branches of the thermomechanical cycle at complex stress history given by the combination of external forces. This makes a qualitative difference with respect to the standard uniaxial loading experiments on SMA polycrystals. What do actually the transformation paths of thermoelastic MT in SMA polycrystal look like at such conditions?

Also the ability of shape memory alloys to "memorize" a particular transformation path after a special thermomechanical treatment, remains evidently the most exciting feature of these materials. The knowledge of transformation paths of SMA polycrystal under externally enforced nonproportional stress conditions is essential for a proper understanding of the mechanics of shape memory effects exhibited by polycrystals of shape memory alloys. In fact, the growing martensite variant particle should be considered to interact with general nonproportional stress even in uniaxial loading of SMA polycrystal [4].

This experimental study has been focused on the progress of MT in SMA polycrystal at variable stress-temperature conditions. We have chosen a combined tension - torsion loading of SMA tubes as a simple and physically well defined test. When the tube is subjected to axial load, $L$, and torque, $M$, the resulting macroscopic stress state can be characterized as average stress across the volume of SMA polycrystal, $\Sigma_{i j}$ in the first approximation. Let us suppose, that the thermoelastic MT is induced by the uniform stress $\Sigma_{i j}$. It has two nonzero components of axial stress, $\sigma$, and torsional shearing stress, $\tau$. Analogically, the average uniform strain, $E_{i j}$, is given by the axial strain, $\epsilon$, and torsional shear strain, $\gamma$. The strain response of SMA polycrystal to the general variation of stress and temperature can thus be systematically examined by independent application of external forces of torque and axial load in combined tension and torsion experiments. The recorded thermomechanical data could serve as a model information about the general stress - strain - temperature behaviors of thermoelastic SMA polycrystal and it is the purpose of this paper to summarize the experimental results obtained.

\section{EXPERIMENTAL}

The combined tension-torsion experiments were performed on tubular specimens made of $\mathrm{Cu}-$ $10 w t . \% A l-5 w t . \% Z n-5 w t . \% M n$ industrial, polycrystalline shape memory alloy (gauge length $\mathrm{l}=35 \mathrm{~mm}(25 \mathrm{~mm}, 40 \mathrm{~mm})$, diameters $\left.d_{e x t}=8 \mathrm{~mm}, d_{i n t}=5 \mathrm{~mm}\right)$. The specimens were finally heat treated at $873 \mathrm{~K}$ for 2 hours and quenched into the ice water (grain size $d \leq 120 \mu \mathrm{m}$ ). Transformation temperatures, found by electric resistivity measurements, are $M_{s}=239 \mathrm{~K}, M_{f}=223 \mathrm{~K}, A_{s}=248 \mathrm{~K}$, $A_{f}=260 \mathrm{~K}$. In thermomechanical loadings, the external stress and temperature were prescribed by the application of axial load and torque in Shimadzu AG-10TS combined load tester. The tubular 


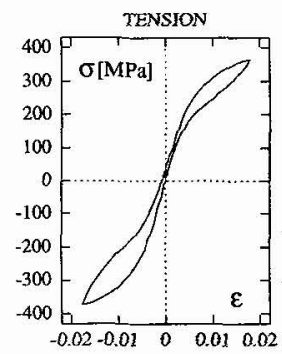

a)

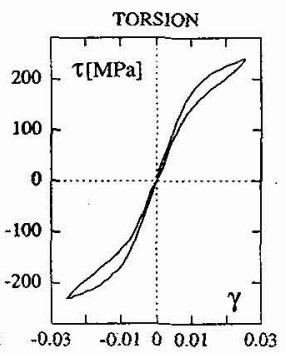

b)

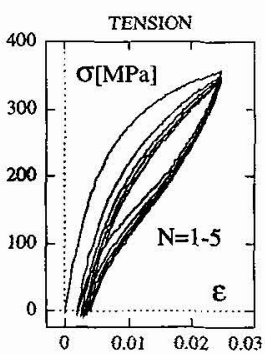

c)

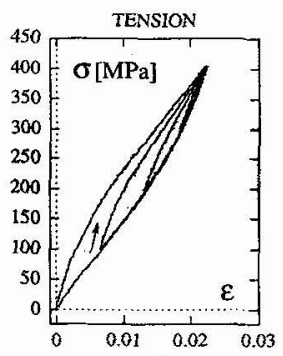

d)

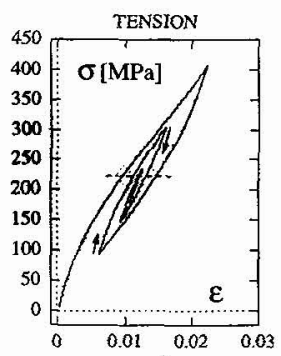

e)

Figure. 2: Uniaxial pseudoelastic stress - strain behaviors (strain control); a) symmetrical tension compression, b) symmetrical torsion+ - torsion-, c) the evolution of the shape of pseudoelastic loop in first 5 cycles, d-e) partial pseudoelastic loops.

specimens were heated/cooled in thermomechanical cycles by hot and cool air $\left(T=285 K\left(A_{f}+\right.\right.$ $25 K)-363 K\left(A_{f}+103 K\right)$ ). Due to a large heat exchange between specimen and grips, a relatively massive specimen and rapid heating/cooling, the precision of the temperature measurement was not good and, therefore, the thermal hysteretic loops will not be presented here. The surfaces of the specimens were electrolytically polished (cyclohexanol and phosphoric acid $3: 2, \mathrm{~T}=313 \mathrm{~K}, \mathrm{U}=6 \mathrm{~V}$, $\mathrm{i}=0.1 \mathrm{~A} / \mathrm{cm}^{2}, \mathrm{t}=600 \mathrm{~s}$ ) and the evolution of surface steps due to stress induced martensite particles was observed in situ by an attached microscope and video recorded.

Due to the difficulties with the evaluation of acting shear stresses and strains from the measured torque - angle, $M-\theta$, dependencies and, simultaneously, the need for a definition of equivalent stress and strain, the following procedure has been adopted. The maximum elastic values of stress and strain on the surface of hollow bar specimens were calculated (3). The yield stresses and strains at which the forward MT starts, were always determined from the first two pseudoelastic loops on each specimen performed in tension $\left(\sigma^{F}, \epsilon^{F}\right)$ and torsion $\left(\tau^{F}, \gamma^{F}\right)$, respectively. The " $0.05 \%$ proof stress" method was employed to determine the yield points. The empirical coefficients $C^{S}, C^{E}(4)$ were calculated for each specimen from the data of the first two loops and used to determine the energetically equivalent values of shear stress, $\tau_{i n v}$, (equation 5) and shear strain, $\gamma_{i n v}$, (equation 5), from the original $\tau$ and $\gamma$ values defined by (3). The equivalent stresses and strains in simultaneous tension - torsion loadings were calculated by equations (6). Such technique to determine the energetically equivalent values of uniform stresses and strains can theoretically be used for elastic strains only. However, our experimental results suggest [13] that it can be successively used for reversible pseudoelastic strains in a good approximation as well. A single equivalent stress - equivalent strain loop can be constructed from the data of combined load tests to the same maximum equivalent stress (strain), regardless of the applied stress (strain) path. This corresponds to the idea that an equal amount of mechanical energy is needed not just to start but also to drive the stress induced MT in torsion and tension deformation modes. It is also in accord with the understanding of transformation pseudoelasticity as a mechanically reversible elastic-like process (the resistive stresses of the transforming material in thermoelastic equilibrium due to the strained matrix and martensite variant interactions are basically of elastic nature). The strain states of the specimen in the combined load tests will be given by $\left[\epsilon, \gamma_{i n v}\right]$ in strain space and their time sequence will be called here "strain path", while the sequence of $\left[\sigma, \tau_{i n v}\right]$ stress states in stress space will be called "stress path". Moreover, the inelastic transformation strains $\epsilon^{t r}$ and $\gamma_{i n u}^{t r}$ are calculated by equations (7) and the time sequence of transformation strain states $\left.\left[\epsilon^{t r}, \gamma_{i n v}^{t r}\right]\right]$ will be called "transformation path" or "transformation pathway".

$$
\begin{array}{cc}
\tau=\frac{16 M d_{e x t}}{\pi\left(d_{e x t}^{4}-d_{i n t}^{4}\right)} & \gamma=\frac{d_{e x t} \theta}{2 l} \\
C^{S}=\frac{\sigma^{F}}{\tau^{F}} & C^{E}=\frac{\gamma^{F}}{\epsilon^{F}} \\
\tau_{i n v}=C^{S} \tau & \gamma_{i n v}=\frac{\gamma}{C^{E}} \\
\sigma_{e q}=\sqrt{\sigma^{2}+\left(C^{S} \tau\right)^{2}} & \epsilon_{e q}=\sqrt{\epsilon^{2}+\left(\gamma / C^{E}\right)^{2}}
\end{array}
$$




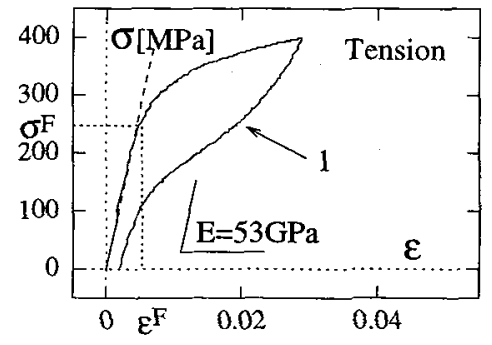

$$
\begin{aligned}
& C^{S}=\sigma^{F} / \tau^{F}=239 / 195=1.23 \\
& C^{E}=\gamma^{F} / \varepsilon^{F}=0.0117 / 0.0053=2.21
\end{aligned}
$$
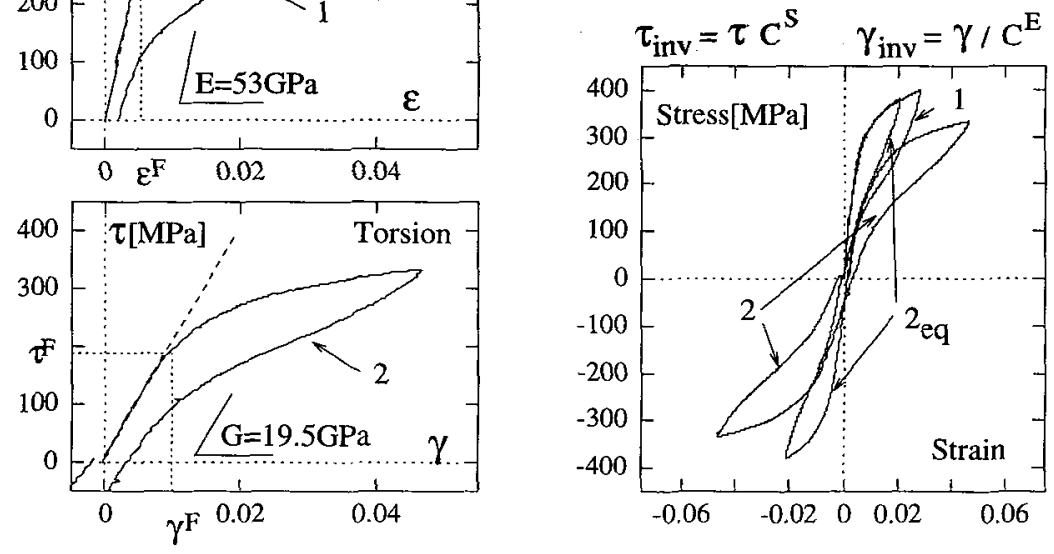

Figure. 3: Uniaxial loading of as quenched specimen; 1..tension, 2. symmetrical torsion $+/-; \epsilon, \gamma, T]$ control; $\mathrm{E}$ and $\mathrm{G}$ are experimentally determined elastic constants in tension and torsion, respectively; $\sigma^{F}, \epsilon^{F}$, are critical stress and strain for forward MT induced in tension $\left(\tau^{F}, \gamma^{F}\right.$ in torsion); $2_{e q}$ loop is the torsion pseudoelastic loop expressed in equivalent values of shear stress and shear strain.

$$
\epsilon^{t r}=\epsilon-\frac{\sigma}{E} \quad \gamma_{i n v}^{t r}=\frac{\gamma}{C^{E}}-\frac{\tau C^{S}}{E}=\frac{1}{C^{E}}\left(\gamma-\frac{\tau}{G}\right)
$$

\section{RESULTS AND DISCUSSION}

Since it is the external stress and temperature, that control the progress of MT, the thermomechanical loadings were performed in stress - temperature $\left[\sigma, \tau_{i n u}, T\right]$ control mode. The time evolution of imposed external stress and temperature throughout the thermomechanical cycle $/ \Sigma-T$ path/ will be graphically presented in 3D $\sigma-\tau_{i n v}-T$ stress-temperature space. Each point on the $\Sigma-T$ path represents an external stress-temperature state of SMA polycrystal. Our study is limited only to a relatively narrow interval of stress - temperature conditions $385 \mathrm{~K}<T<423 \mathrm{~K}$ and $\sigma_{e q}<500 M P a$, where the alloy is supposed to exhibit transformation pseudoelasticity. Starting from the stress free, room temperature $[0,0,285]$ stress-temperature state, various paths can be imposed as schenatically suggested in Figure 1a. The experimental data of the thermomechanical experiment in the format $[\mathrm{t}, \mathrm{T}, \epsilon, \sigma, \gamma, \tau]$ have necessarily a tensorial character and are difficult to represent in a complete and easily readable form. We will use $2 \mathrm{D}$ sections through the data format for graphical illustration of the information obtained.

\subsection{Uniaxial loading at constant temperature}

The thermoelasticity of $\mathrm{Cu}-\mathrm{Al}-\mathrm{Zn}-\mathrm{Mn}$ SMA polycrystal as a model material can be well characterized by uniaxial pseudoelastic loops at room temperature (Figure 2) and thermal dependence of critical stress for forward MT (Figure 1b). While the torsion + - torsion- pseudoelastic loops in stabilized state are always perfectly symmetrical, the loops in compression exhibit typically a larger hysteresis width in comparison to the tensile loop. Nevertheless, the forward branches of both loops are well symmetrical in spite of the expected asymmetry of transformation yield surfaces of SMA single crystals exposed to general stresses [8]. The shape of pseudoelastic loops changes in a few introductory mechanical cycles (Figure 2c), but becomes stabilized and remains almost unchanged for a large number of cycles. The partial pseudoelastic loops (Figure $2 \mathrm{~d}, \mathrm{e}$ ) in mechanically stable state characterize 
the path dependency and dissipative nature of thermoelastic MT. The linearization of the temperature dependence of equivalent stress for the start of forward MT should be understood as a first approximation and guide to the thermomechanical data. The proportionality constant $s=3.3 \mathrm{MPa} / \mathrm{K}$ is larger than measured on single crystals of similar Cu-based SMA ( $\mathrm{s}=1.67$ for Cu-Al-Mn [9], $\mathrm{s}=1.25$ for $\mathrm{Cu}-\mathrm{Al}-\mathrm{Zn}[10])$. Such differences are well known and were recently studied by Ono [11] in the frame of modified Taylor model for deformation of polycrystals.

COMBINED

TENSION - TORSION

$[\sigma, \tau, \mathrm{T}]$ CONTROL

$$
\begin{aligned}
& C^{S}=1.23 \\
& C^{E}=2.2
\end{aligned}
$$

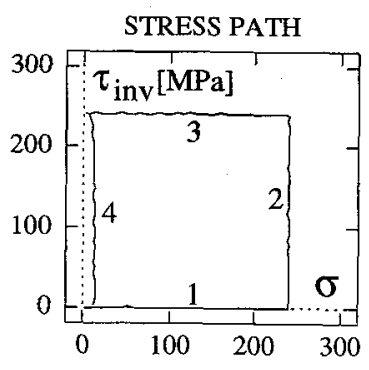

TORSION
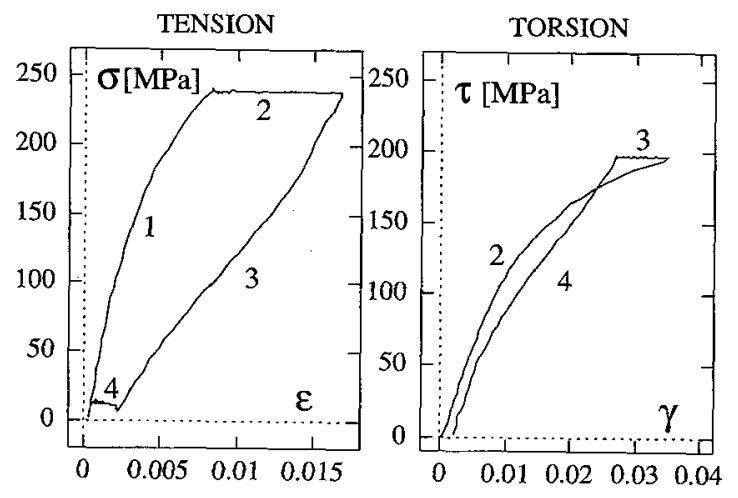
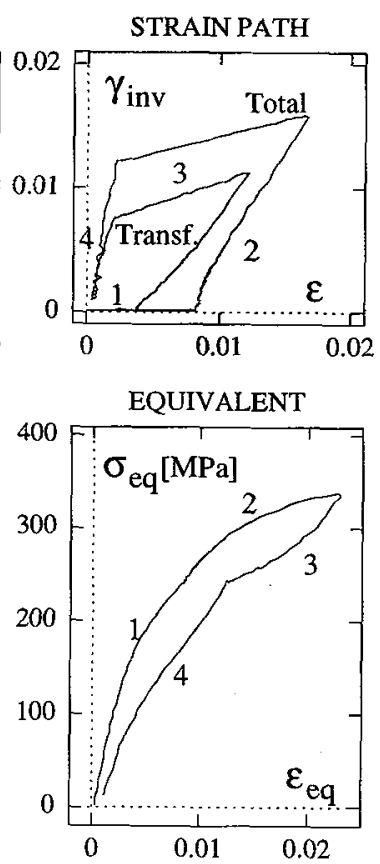

Figure. 4: Nonproportional combined load test in stress control; the subsequent stages of the test are numbered 1-4; both strain path (Total) and transformation path (Transf.) are drawn in strain space.

Due to the changes of the shape of pseudoelastic loop with number of cycles, only the first tests in each deformation mode on as quenched specimens bring a meaningful information about the critical stress states $\left[\sigma^{F}, \tau^{F}\right]$ to induce the forward MT at constant temperature. Such two pseudoelastic loops, performed on a single specimen, are shown on Figure 3 . The shear stress, $\tau$, and shear strain, $\gamma$, represent the maximum values on the surface of still a relatively thick wall tube and are not fully appropriate for the description of the strain and stress states of the transforming system as a whole. Due to this uncertainty and, at the same time, due to the need for a definition of equivalent stress but lacking the knowledge about yield and flow criterion for SMA polycrystal, we introduced directly the energetically equivalent values of shear stress, $\tau_{i n v}$, and shear strains, $\gamma_{i n v}$, in torsion. If the torsion pseudoelastic curve is drawn in $\tau_{i n v}, \gamma_{i n v}$ coordinates $\left(2_{e q}\right.$ loop $)$, the density of phenomenologically evaluated elastic energy, necessary to trigger $M T$, is by definition equal in tension and torsion tests. However, as we can see on Figure 3 , not only the critical stresses and strains coincide, but also the whole forward branches of both pseudoelastic loops recorded in tension and torsion automatically fit one another as a result. The character of $\sigma-\epsilon$ and $\tau-\gamma$ pseudoelastic loops changes essentially in a few subsequent pseudoelastic cycles (see Figure 2a,b). Nevertheless, the once determined coefficients for equivalent stresses and strains results in a very good fit of the whole tensile and torsional pseudoelastic loops in stabilized state, if the same maximum equivalent stress (strain) is imposed. In order to bring the specimen into a relatively stable mechanical state, together at least 10 introductory loops in all deformation modes used were necessary. When the introductory loops were performed along the same (similar) strain path, an essential strain anisotropy was introduced into the specimen microstructure. Consequently, a measurable nonproportionality among stress and strain paths was observed in subsequent deformation tests as reported in [12]. 

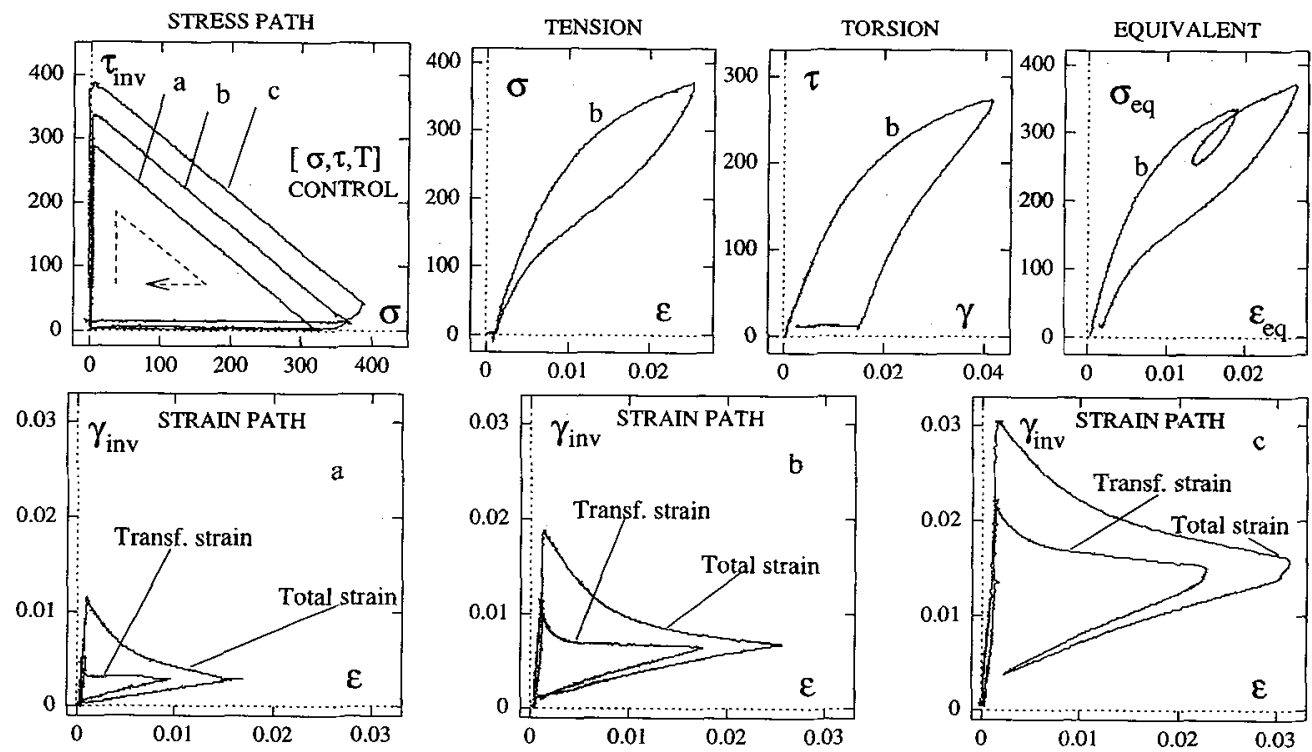

Figure. 5: Reloading of partially transformed SMA polycrystal; triangular stress path a,b,c; both the strain path (Total) and transformation path (Transf.) are drawn in strain space.

\subsection{Combined tension-torsion loading at constant temperature}

When the SMA polycrystal is subjected to the proportional loadings at constant temperature using simultaneous tension-torsion $\frac{\Delta \tau}{\Delta \sigma}=$ const. $\left(\frac{\Delta \gamma}{\Delta \epsilon}=\right.$ const.), the strain (stress) response of the specimen is really proportional to the imposed external stresses (strains). The results of such experiments can be found in [13].

A qualitatively new information about MT can be obtained from nonproportional experiments (for example torsion at applied axial force), in which the uniform stress changes in a nonproportional way. The stress induced martensite variant particles need not to be the most favored ones following a nonproportional stress change, stop to grow, interact with other newly nucleated particles or even shrink back to the parent phase. What is the macroscopic stress - strain behavior of SMA polycrystal at such loading conditions, has been systematically studied by nonproportional combined load tests.

The stress path is imposed in a stress control test (Figure 4) and the recorded strain response of the specimen is illustrated by the separate pseudoelastic loops $\sigma-\epsilon$ and $\tau-\gamma$, strain path and transformation path in strain space and equivalent stress - strain, $\sigma_{e q}-\epsilon_{e q}$, loop. The first important result is, that the pseudoelastic strains in a nonproportional loop are again completely reversible. The shape of $\sigma-\epsilon, \tau-\gamma$ pseudoelastic loops becomes rather exotic, since they separately contain only part of the information about the stress - strain constitutive behavior, expressed by equation 1 . On the other hand, the equivalent stress - strain $\sigma_{e q}-\epsilon_{\mathrm{eq}}$ curve still fits the shape of uniaxial loadingunloading pseudoelastic loop to the same maximum equivalent stress. The $\sigma_{e q}-\epsilon_{e q}$ loop characterizes the mechanical energy stored in each stage of the nonproportional test (compare Figures 3 and 4 - different specimens, similar maximum equivalent strains). If we consider the area enclosed by $\sigma_{\varepsilon q}-\epsilon_{e q}$ loop (or better the sum of areas enclosed by $\sigma-\epsilon, \tau_{i n v}-\gamma_{i n v}$ separate loops) as a measure of the total dissipated energy in a nonproportional loop, we observe, that the energy dissipation in the nonproportional test is not any larger than in the proportional one. This justifies the usage of equations 5, 6 even for nonproportional tests. The shape of recorded strain paths documents a coupling among tension and torsion under stress not only in forward MT (as typically observed in metal plasticity and described in plasticity theory by yield criterion and flow rule for loading) but also on unloading due to the reverse MT. Additional experimental results can be found in [13, 14].

In another experiment, the strain responses of partially transformed SMA polycrystal to the 


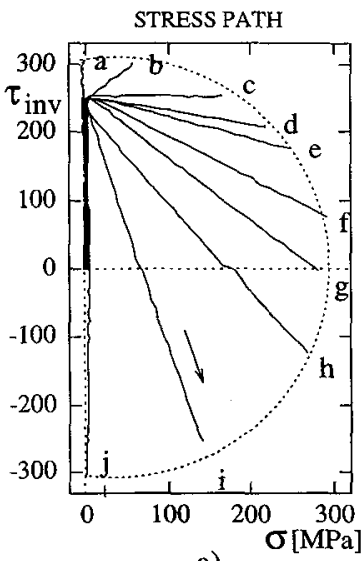

a)

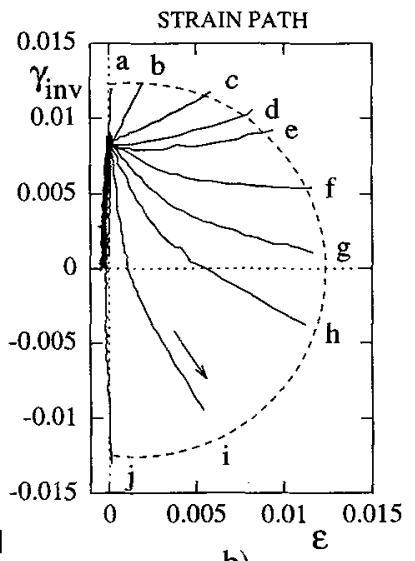

b)

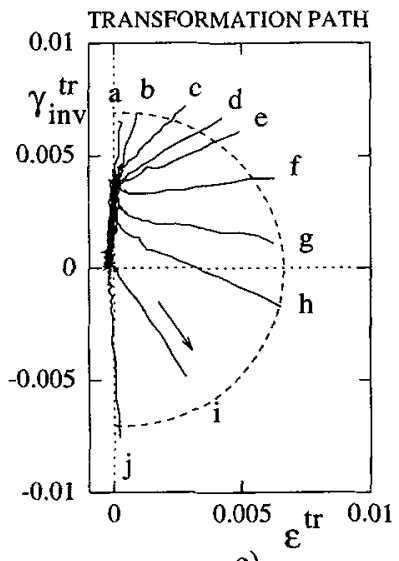

c)

Figure. 6: The strain response of partially transformed SMA polycrystal to the change of stress paths along directions a - $\mathbf{j}$; maximum equivalent stress $\sigma_{e q}^{M}=300 \mathrm{MPa}$. a) imposed stress path, b) strain paths, c) transformation paths

reloading between two maximum stress states for 3 different stress paths were examined (Figure 5). Reloading proceeds at stress levels increasing from path a to path c. The strain paths as well as transformation paths are shown for all 3 tests, while stress-strain response is presented only for test $b$. At low stresses (path a), the transformation strain response of SMA polycrystal on reloading can be clearly divided into 2 separate phases - 1) reverse transformation along the already passed forward transformation path followed by 2) forward MT under new stress direction. However, the reverse MT is by no means completed, when the forward MT starts $\left(70 \%\right.$ of $\gamma_{i n v}^{i r}$ remains unrecovered, when reloading is finished). The strain responses are different at medium stresses (paths $b, c$ ). There is a transition region between the reverse and forward MT that widens with increasing level of stress on reloading (increasing fraction of transformed martensite phase). It should be noted, that the reloading branch of this type of nonproportional test is described by the partial pseudoelastic loop on $\sigma_{e q}-\epsilon_{e q}$ curve in accord with the energetical argumentation above.

Special experiments were performed to study systematically the strain response of the partially transformed SMA polycrystal to the various general stress changes. The results are shown on Figure 6. The specimen was deformed 10 times, at first in torsion up to the stress state $\left[\sigma, \tau_{\text {inv }}\right]=[0,250]$ and then the loading continued further along 10 various stress paths a-j up to the maximum equivalent stress, $\sigma_{e q}^{M}=300 \mathrm{MPa}$ (Figure 6a) (only forward MT paths are shown here, and the $\sigma_{e q}^{M}$ is slightly smaller than $300 \mathrm{MPa}$ in cases $\mathrm{g}$ and $\mathrm{i}$ due to control errors). The recorded evolution of macroscopic strains - strain paths (Figure 6b) and average transformation strains - transformation paths (Figure $6 \mathrm{c}$ ) provide a systematic information about the behavior of stress induced martensite, subjected to the various types of general stress changes. At first, it should be noted that, regardless of the shape of applied stress paths a-j, the maximum strain states, $\left[\epsilon^{M}, \gamma_{i n y}^{M}\right]$, corresponding to the maximum equivalent stress $\sigma_{e q}=300 M P a$ are again located near the half circle in strain space. This is not true for transformation strains, particularly, the maximum transformation strains corresponding to the $c, d$,e and $f$ stress paths. The transformation strains develop partially further in torsion + after the change of stress path direction in $a, b, c$. This would be largely expected, since the mechanical work continues to be supplied $\frac{d \sigma_{e q}}{d t}>0$ after the stress path direction change. The reverse transformation clearly starts after the change of stress path direction in tests e,f,g,h,i,j $\left(\Delta \gamma_{i n v}^{t r}<0\right.$ and $\Delta \epsilon^{t r}=0$ ), but it is completed only in cases $\mathrm{i}, \mathrm{j}(\mathrm{h} ?)$. Do the martensite variant particles, nucleated in the beginning torsion + branch of the applied stress path, shrink, when the mechanical work is being again supplied on reloading along stress paths $\mathrm{g}, \mathrm{h}$ ? Is there a reorientation of stress induced martensite particles or the reverse transformation stops uncompleted and new martensite variant particles are nucleated and grow, causing thus the decrease of the measured torsion+ transformation strain component, $\gamma_{i n v}^{t r}$ ? What happens on the microlevel of transforming martensite variant particles remains a theme for further experimental and theoretical investigations. 
a)

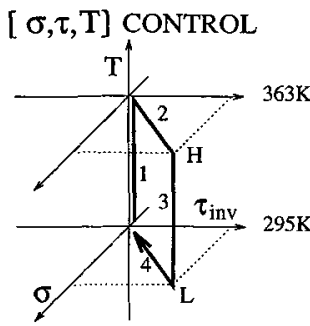

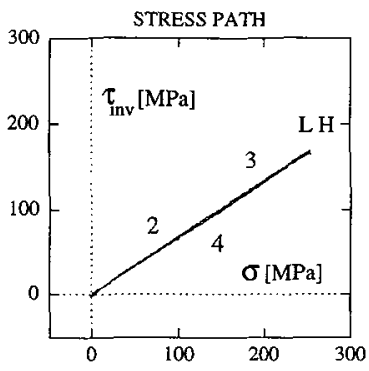
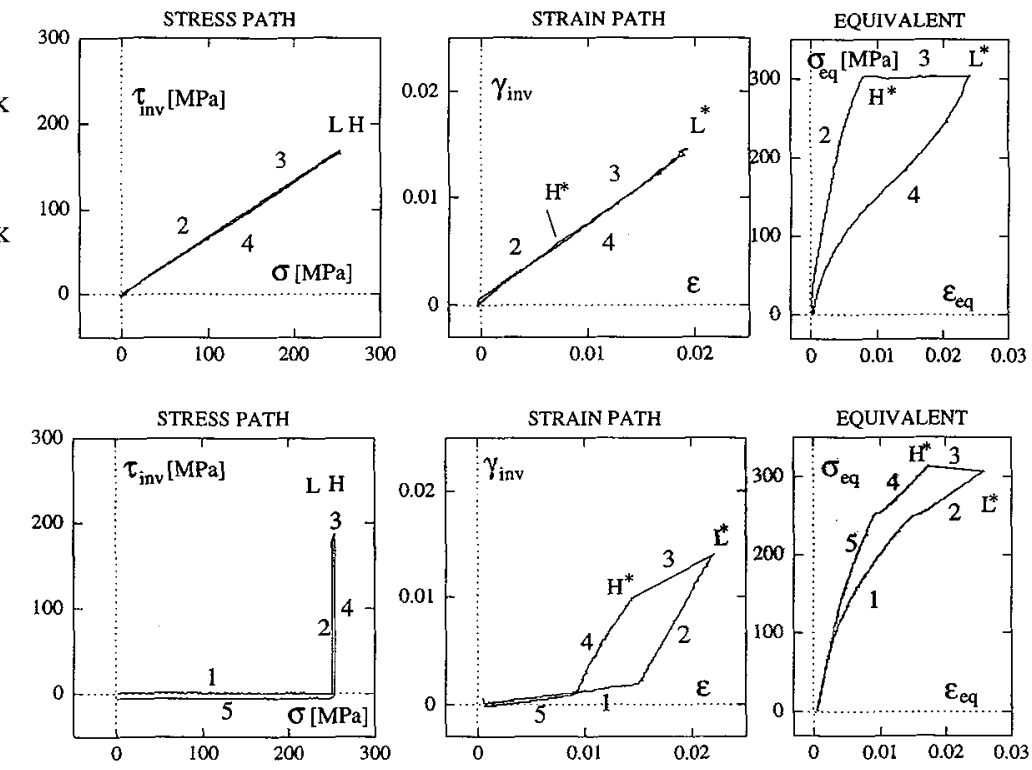

EQUIVALENT
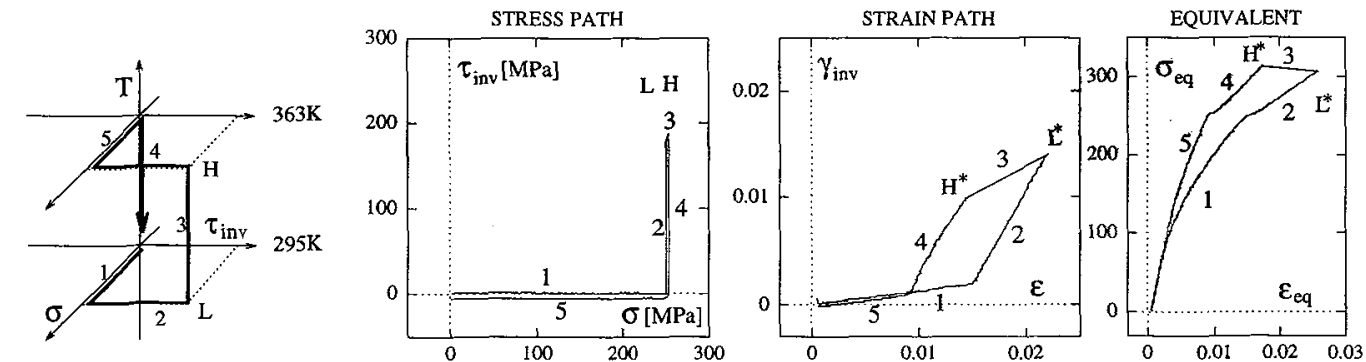

Figure. 7: Thermomechanical tests with SMA polycrystal; a) proportional, b) nonproportional; the stress-temperature state $\mathrm{L}=\left[\sigma, \tau_{i n v}, T\right]=[250,180,295]$ is reached along different stress - temperature paths in $\mathrm{a}$ and $\mathrm{b}$

\subsection{Combined tension-torsion-temperature thermomechanical loading}

Since thermoelastic martensitic transformation can be induced by the stress as well as temperature, the constitutive description of SMA polycrystal has to be necessarily a thermomechanical one. Macroscopic thermomechanical data for SMA polycrystals are available for uniaxial stress conditions and wide temperature range including thermal cycling at constant stress or strain[15] or shape memory effects $[16,17]$.

Thermomechanical cycling of SMA polycrystals, in which the applied stress varies in a nonproportional way can be performed by combined tension and torsion technique. The results of 2 thermomechanical cycles are shown on Figure 7, a) proportional thermomechanical test, b) nonproportional thermomechanical test. The stress-temperature state $\mathrm{L}=[250,180,295]$ has been reached by continuous forward MT in both cases (path segments 1-2-3 in 7a and 1-2 in 7b). The strain states $L^{*}$, as a specimen responses, are similar in tests $\mathrm{a}, \mathrm{b}$, in spite of the relatively large inelastic strain $\approx 1.5 \%$. This is not the case for high temperature strain states state $H^{*}$. The reason is a larger energy dissipation in the test $b$, in which the stress - temperature state $H=[250,180,363]$ has been reached along both forward and reverse transformation paths, compared with the forward transformation path only in the test a.

While the progress of forward MT on cooling seems to be proportional to the applied stress state, regardless of the previous deformation history, the reverse MT on heating deserves a special attention. If the specimen is deformed in a nonproportional way and heated at constant maximum stress state. (see Figure 8), the simultaneous tension-torsion strain reversal is observed. The transformation path is a linear path in the strain space between the maximum transformation strain state $\left[\epsilon^{t r}, \gamma_{i n v}^{t r}\right]$ and the origin $[0,0]$. As a result, the external strain state of SMA polycrystal changes from the low temperature state $L^{*}$ along a linear strain path in the direction towards the elastic strain state, that corresponds to the applied stress.

Finally, the data of a more complex thermomechanical cycle are shown on Figure 9. Two heating/cooling subcycles at constant stress were performed during the nonproportional mechanical test. The responses of SMA polycrystal to heating/cooling are qualitatively different, if the thermal subcycle is performed at constant maximum stress state D (reached along continuous forward transformation path) or general stress state as $\mathrm{E}$. While the transformation strains, recorded during heating/cooling subcycle in stress state D, are almost reversible, the strain paths are different during heating and cooling branches of the thermal subcycle in the general stress state E. These results 

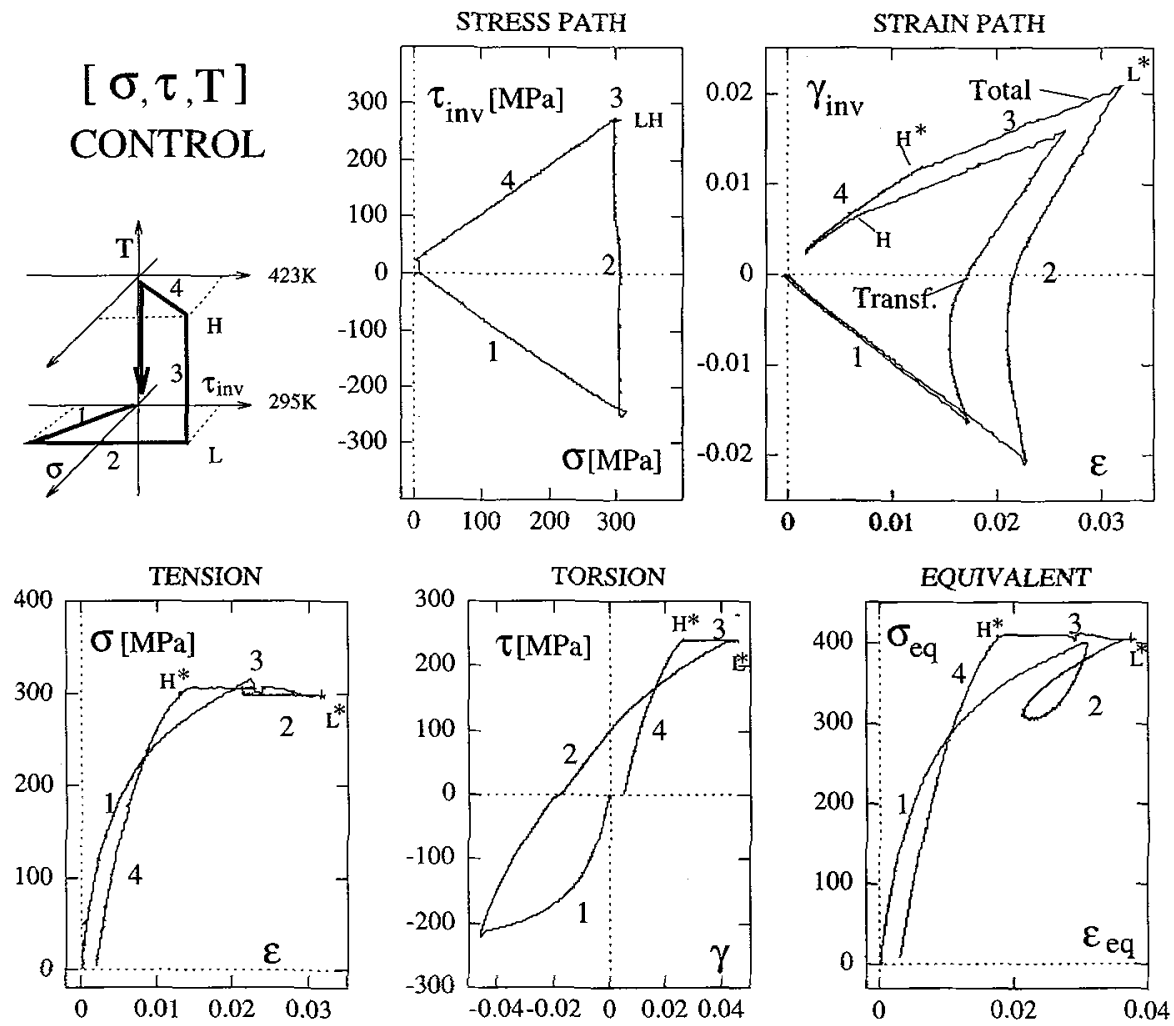

Figure. 8: Nonproportional thermomechanical test; reverse transformation by heating at constant stress; both strain path (Total) and transformation path (Transf.) are drawn in strain space.

clearly show the effects of energy dissipation in nonproportional thermomechanical loading of SMA polycrystal.

The detail analysis of the results of in situ observations of specimen surface is out of the scope of this paper, nevertheless a few basic characteristics are worth of mentioning here. The surface steps, that correspond to the stress induced martensite particles in surface grains, appear in the forward and disappear in the reverse branches of the pseudoelastic loop. The development of surface step pattern is highly inhomogeneous. In an arbitrarily chosen grain, the steps of different geometry appear in different deformation modes (tension, compression, torsiont and torsion-). However, the identical steps repeatedly appear in the same deformation mode. While in proportional tests (for example simple loading - unloading in tension), the steps regularly disappear in reverse order they were created, this is not, necessarily, the case for nonproportional pseudoelastic cycles. The steps, that were created in the beginning of forward MT, need not be the last to disappear on unloading, if forward and reverse transformation paths are different.

The experimental data presented refer actually only to a single SMA material and narrow interval of external stress - temperature conditions. The pseudoelastic loops were limited to lower stresses (low fraction of transformed volume of martensite phase). The stress states near biaxial tension, biaxial compression or triaxial stress can not be simulated by combined tension and torsion. We also do not have reliable thermomechanical data for temperatures near or below the martensite start temperature including one way shape memory effect. Nevertheless, as an abstraction of the above experimental results, the transformation start surface for forward MT of SMA polycrystal can be drawn in $\Sigma-T 3 D$ space (see Figure 1a) in the form of half cone surface as a first approximation. Such cone surface contains the experimental critical stress-temperature states $\left[\sigma, \tau_{i n v}, T\right]^{F}$ to induce forward MT. The circular "yield surface" for mechanical loading at constant temperature does not automatically imply, that the material obeys the Von Mises yield criterion, because of the definition of $\tau_{i n v}$ in torsion (the $C^{S} \neq \sqrt{3}$ ). The distance of a stress point on the cone surface from the vertical T-axis characterizes the mechanical work necessary to trigger MT at given temperature. The strain 
response of pseudoelastic SMA polycrystal to the change of external forces along the prescribed $\Sigma-T$ path is well defined and reproducible along the same path.
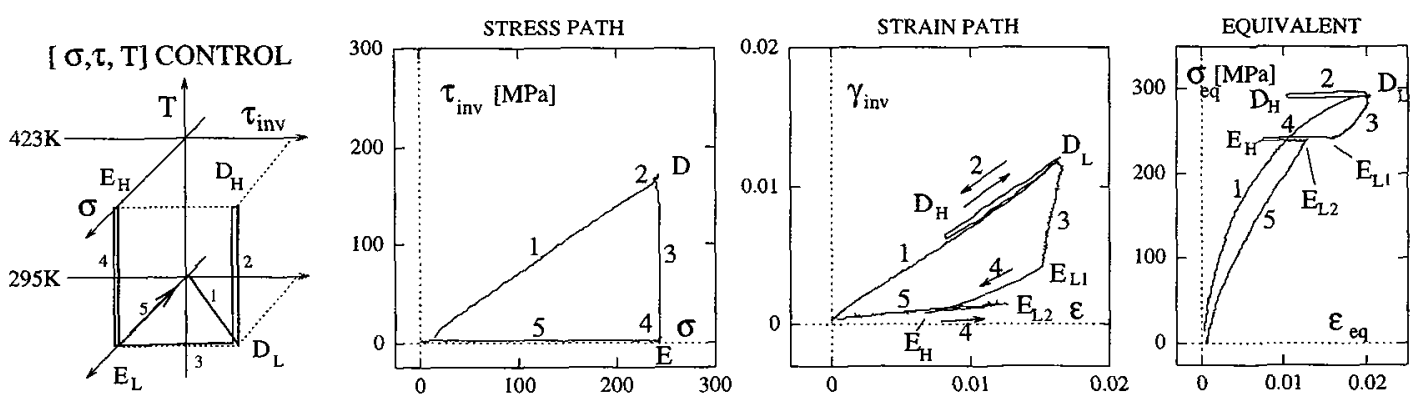

Figure. 9: Nonproportional thermomechanical test; thermal cycling at constant stress states D, E

\section{CONCLUSIONS}

The progress of stress induced thermoelastic martensitic transformation under general stress conditions has been studied in thermomechanical loading experiments by combined tension-torsion of tubular specimens of $\mathrm{Cu}-\mathrm{Al}-\mathrm{Zn}-\mathrm{Mn}$ SMA polycrystals at variable temperature.

- It has been shown, that the SMA polycrystal can be loaded - unloaded repeatedly by the change of temperature and general combination of external forces of axial load and torque with relatively well defined, path dependent but well reproducible strain path response.

- The transformation paths - the measured evolutions of average transformation strains in tensiontorsion strain space in response to the prescribed stress temperature history - were presented.

\section{Acknowledgements}

The support by grants of Japanese Ministry of Education and Iketani Science Foundation is greatly acknowledged.

\section{References}

[1] Otsuka K., Shimizu K., Int. Met. Rev. 31 (1986) pp.93-114

[2] Wollants P., Roos J.R. and Deleay L., Progress in Material Science 37 (1993) pp.227-288.

[3] Kato M. and Pak H. Phys. Stat. Sol. (b) 123 (1984) pp.415-424.

[4] Ping Xu and Morris J.W., Metall. and Mater. Trans. 24A (1993) pp.1281-1294.

[5] Tanaka K., Oberaigner E.R. and Fisher F.D., AMD-Vol. 189 Mechanics of Phase Transformations and Shape Memory Alloys 292 (1984) ASME pp.151-157.

[6] Patoor E., Eberhardt A. and Berveiller M., Pitman Research Notes in Math. Series 296 (1993) pp.38-j4.

[7] Marketz F. and Fisher F.D. Metall and Mater. Trans. 26A, (1995) pp.267-278.

[8] Buchheit T.E. and Wert J.A., Metall. and Mater. Trans. 25A (1994) pp.2383-2389.

[9] Kato H., Dutkiewicz J. and Miura S. Acta Metall. 42 (1994) pp.1359-1365.

[10] Pelegrina J.L. and Ahlers M. Acta Metall 40 (1992) pp.3205-3211.

[11] Ono N. and Sato A., Trans. of Jap. Inst. Met. 29 (1988) pp. 267-273.

[12] Sittner P. and Tokuda $M$ in "Advanced Materials 93' V/B: Shape Memory Materials and Hydrides, ed. K.Otsuka et al., Trans., Mat., Res., Soc., Jpn., 188B (1994) pp,951-955.

[13] Sittner P., Hara Y. and Tokuda M., "Pseudoelastic Deformation in Combined Tension and Torsion", ICSMA-10, August 21-26, 1994, Sendai, Japan, ed. Oikawa H. and Maruyama K., pp.319-322.

[14] Sittner P., Hara Y., and Tokuda M., "SMA hysteresis under combined tension and torsion", Shape Memory Materials-94, September 25-28, 1994, Beijing, China, ed. Hsu T., pp. 541-545.

[15] Sawada T., Tobushi H., Kimura K., Hattori K., Tanaka K. and Lin P., JSME International 